\title{
EL DERECHO INTERNACIONAL COMO OBJETO DE LOS ESTUDIOS INTERNACIONALES*
}

\author{
María Teresa Infante $C$.
}

Los estudios internacionales es un área dominada fundamentalmente por el tema de la política exterior. De allí que en este trabajo se definen en primer lugar los objetivos centrales de la enseñanza del Derecho Internacional. Al mismo tiempo, para ayudar a comprender mejor su función y especificidad, se describen tres niveles de acción diferentes de las relaciones internacionales donde esta materia se desenvuelve: el sistema mundial global y los problemas de paz y seguridad; la relación entre el orden jurídico y la realización de los intereses nacionales de los Estados; y la interdependencia entre Estados y su relación con otros actores. Finalmente, teniendo en cuenta lo anterior, se refiere a los programas y contenidos que debe contemplar la enseñanza actual de esta disciplina, en un marco que presente los escenarios reales de las relaciones jurídicas internacionales e incorporando nuevos enfoques.

Esta reflexión se sitúa en el marco de la Década del Derecho Internacional, proclamada por la $46^{\mathrm{a}}$ Asamblea General de las Naciones Unidas e intenta plantear elementos centrales para la comprensión de la disciplina dentro de los estudios internacionales.

El objeto principal de la enseñanza del Derecho Internacional y su investigación, es hacer transparente el concepto del orden jurídico internacional, su ámbito de aplicación, las condiciones para la efectividad de sus principios, así como permitir una comprensión amplia del proceso de desarrollo y transformación de sus instituciones. Todo ejercicio académico debe tener entre sus propósitos, asimismo, la presentación clara de las funciones reales de este Derecho.

En otros términos, lo que Friedmann denominó hace años "la estructura cambiante del Derecho Internacional", ${ }^{1}$ debe estar pre-

\footnotetext{
*Simposio "Enseñanza, Estudio, Difusión y Apreciación amplia del Derecho Internacional en los países en Desarrollo", convocado por el Departamento Jurídico y de Tratados del Ministerio de Relaciones Exteriores de China, Beijing, 24-26 de agosto de 1992.

${ }^{1}$ Friedmann, W., The Changing Structure of International Law, (New York: Columbia University Press, 1964).
} 
sente en los enfoques que muestran la vinculación del Derecho Internacional con el sistema internacional en el cual opera. ${ }^{2}$

Tendrá que considerarse al mismo tiempo, que el trabajo académico significa un ejercicio racional que no siempre coincide con la creación efectiva y la aplicación o ejecución de principios e instituciones del Derecho Internacional. Debe hacerse, en consecuencia, una clara distinción entre los postulados doctrinales y los principios y reglas que en la práctica gozan de eficacia, observándose su funcionamiento en procesos de negociación, de resolución y de adjudicación por los órganos internacionales.

Para analizar con mayor profundidad el tema propuesto, debemos considerar tres niveles diferentes en las relaciones internacionales, cuyo conjunto ofrece un panorama más completo de las funciones del Derecho Internacional.

\section{Niveles de acción del Derecho Internacional}

a) El concepto de sociedad mundial o globaly los problemas de pazy seguridad, constituye el primer nivel para situar el problema.

Es importante considerar sobre este punto, la influencia que han tenido y continúan teniendo los enfoques doctrinales acerca de la naturaleza de la sociedad en que opera el Derecho Internacional. ${ }^{3}$ Los idealistas sostienen que la paz sólo puede alcanzarse mediante el Derecho, lo cual es abiertamente discutido por los realistas que niegan al Derecho ese poder, privando de todo contenido jurídico a los esfuerzos para alcanzar arreglos de paz. La sumisión total del ordenamiento jurídico a las relaciones de poder o a las características del sistema internacional, será una consecuencia esencial de esta última teoría.

En los últimos años se ha postulado por una superpotencia que ha nacido un Nuevo Orden Mundial, basado en el cumplimiento del Derecho Internacional ${ }^{4}$ junto con otros requisitos de naturaleza política.

\footnotetext{
${ }^{2}$ Carrillo Salcedo, J, A., El Derecho Intemacional en un mundo en cambio, (Madrid: Tecnos, 1984).

${ }^{3}$ De Visscher, Ch., Théories et rélites en Droit Internażional Public, (Paris: A. Pédone, 1970), $4^{2} \mathrm{ed}$.

4 "El concepto de nuevo orden mundial del Presidente Bush", Temas de Política Internacional, Servicio Informativo Embajada de los Estados Unidos de América, 10 de junio de 1991.
} 
Sin calificar el contexto histórico de este enunciado, que coincide con el inicio de la post Guerra Fría, puede concordarse que hay serias evidencias de una necesidad de fortalecer el rol dembecho Internacional a fin de salvaguardar fines básicos de estabilidad, coexistencia y cooperación dentro de la sociedad internacional: Puede sostenerse en este sentido, que en la medida en que las rè glas jurídicas son efectivas, operan como un factor de paz, dado que facilitan la prevención de colisiones entre las distintas políticas seguidas por diferentes Estados.

Debido a que una de las mayores críticas que se hacen sobre esta materia al Derecho Internacional es su falta de organización supraestatal y carencia de ejecutoriedad mediante el recurso a la fuerża, resulta de interés plantear un enfoque que privilegie más bierı el punto de vista de si ese orden jurídico se refleja en las políticas de los Estados y en las relaciones entre ellos, ${ }^{5}$ lo cual requiere de conceptos menos teóricos y más basados en la práctica diplomática.

En este sentido, los enfoques jurídicos operan mezclados con los políticos y permiten una guía más precisa en el esfuerzo de búsqueda de acuerdos en el marco de negociaciones internacionales. Por otra parte, debe considerarse que existe un sitio especial para el Derecho Internacional si las organizaciones internacionales son llamadas a jugar un papel decisivo en los asuntos de seguridad internacional en el nivel universal o regional.

No sólo el proceso de adopción de decisiones relacionado con este tema requiere de consideraciones legales, sino además la determinación e interpretación de los principios a ser aplicados mediante ese sistema. El documento del Secretario General de la onv, Boutros-Ghali, ${ }^{6}$ constituye una propuesta definitiva en este sentido. Corresponde a una intención semejante el tratamiento del tema de la "intervensión humanitaria" dentro del marco de la seguridad colectiva conforme al Capítulo VII de la Carta de la onU, una de cuyas bases legales está dada por las obligaciones erga omnes de respeto a los Derechos Humanos y las que derivan del Derecho Internacional Humanitario.

b) La interacción entre el orden jurídico y la realización de intereses nacionales que se manifiestan en las relaciones entre dos o

\footnotetext{
${ }^{5}$ Henkin, L., How Nations Behave, (New York: Council on Foreign Relations, Columbia University Press, 1979), pp. 22-27, $2^{\mathrm{a}}$ ed.

${ }^{6}$ Boutros Boutros-Ghali, Un panorama de paz, (Nueva Yotk: Naciones Unidas, 1992).
} 
más Estados, constituye otro nivel de interés. Sin duda, el Derecho Internacional tiene una función central en la garantía de la estabilidad de esas relaciones y en proporcionar medios para la solución pacífica de las tensiones y disputas.

La lógica de este sistema opera de manera tal que los Estados muestran voluntad en someterse a obligaciones internacionales y en apoyar el funcionamiento de éstas. Hay que comprender que, si bien las controversias no tienen siempre un origen jurídico y que el proceso de solución no consiste en una simple operación jurídica, las consideraciones jurídicas son indispensables para alcanzar cualquier acuerdo que permita conducir las relaciones entre Estados a un mayor nivel de cooperación.

Sin necesidad de crear un orden imperativo supranacional como en el nivel de análisis que previamente se explicó, la solución de controversias entre Estados constituye una tarea de importancia para el Derecho Internacional. Lo es también la diplomacia, mediante las diversas funciones que este ordenamiento jurídico posee en la política exterior del Estado.

A pesar de que la relación entre el Derecho Internacional y la política exterior puede llevar a consideraciones favorables a un relativismo de sus normas, resulta útil concebir algunas de sus funciones en el comportamiento de los Estados, ${ }^{7}$ como las de determinación de la conducta, la justificación y legitimación de una opción previamente adoptada y, la organización de las relaciones internacionales.

Las relaciones interestatales no carecen de problemas y diferencias en la determinación e interpretación del contenido exacto de las obligaciones internacionales. Un ejemplo reciente se encuentra en la opinión de la Corte Suprema de Estados Unidos en el caso Humberto Alvarez Machain (1992), ${ }^{8}$ la cual establece que los principios generales del Dérecho Internacional no constituyen una base para interpretar un Tratado de extradición en el sentido de que prohibe los secuestros internacionales y, en consecuencia, el hecho del secuestro forzado del demandado no impide su enjuiciamiento por un tribunal de Estados Unidos por violación del Derecho Penal de ese país. La Corte señaló asimismo, que no le correspondía determinar si la acción de Estados Unidos violaba el Derecho Internacional general.

\footnotetext{
${ }^{7}$ Lacharrière, G., de La Politique Juridique Extéricure, (Paris: Economica, 1983), pp. 204-206.

${ }^{8}$ Supreme Court of the United States. N $91-712$, United States, Petitioner v. Humberto Alvarez - Machain on writ of certiorari to the United States Court of Appeals for the Ninth Circuit (June 15, 1992).
} 
Como ha ocurrido en otras ocasiones similares, esta opinión puede generar tendencias hacia una mayor clarificación de las obligaciones que imponen los tratados de extradición que influencien futuras conductas de los Estados exactamente en la dirección opuesta a la interpretación apoyada por el alto tribunal.

Por otra parte, los regímenes internacionales negociados por los Estados proporcionan un cuadro complementario acerca de la función del Derecho Internacional en este ámbito: Es el caso del Sistema del Tratado Antártico, basado en el Tratado de 1959, el régimen del espacio exterior, o la emergencia del nuevo Derecho del Mar y la creación de nuevos espacios marítimos a partir de los años setenta en adelante. En estos casos, el Derecho Internacional ha servido de marco básíco de acomodación y arreglo de reclamaciones, intereses legítimos, derechos y deberes entre diferentes sujetos. Será así el caso también de las negociaciones fundamentales en materia de protección del medio ambiente, como las relativas a la cápa de ozono, la biodiversidad y el cambio climático.

c) La interdependencia entre Estados y su relación con otros actores de la vida internacional, constituye un tercer nivel de análisis.

La soberanía del Estado está sometida desde hace décadas a las limitaciones de la interdependencia y el reconocimiento del hecho de las relaciones transnacionales y la expansión mundial de ciertos valores atribuidos a los individuos. Su impacto mayor ha sido la necesidad de adaptar el ordenamiento jurídico interno a los principios e instituciones internacionales que han emergido crecientemente desde la segunda post guerra.

Las relaciones económicas internacionales han demostrado asimismo, una gran capacidad para contribuir a este respecto. Por ejemplo, en América Latina, después de un largo período de rehusar la aceptación de instrumentos internacionales para la solución de las controversias relativas a inversiones extranjeras, algunos de estos países han llegado a ser Parte en la Convención del Banco Mundial sobre arreglo de diferencias relativas a inversiones, o adoptado tratados de protección de inversiones con otros Estados de la región. ${ }^{9}$ Es el caso de Chile y Argentina, cuando firmaron en 1991 un tratado

\footnotetext{
${ }^{9}$ Jiménez de Aréchaga, E., "La protección đel inversor en el Derecho Internacional", en: $\mathrm{R}$ Vinuesa (ed,), Temas de Derecho Intemacional, (Buenos Aires: Fundación del Centro de Estudios Internacionales de Buenos Aires, 1989), pp. 53-60.
} 
que establece al arbitraje internacional para sus controversias relativas a inversiones que se realicen en el marco de ese instrumento. ${ }^{10}$

Un ejemplo de este tipo de problema jurídico, es la búsqueda de un conjunto de principios para proteger el medio ambiente según el concepto del desarrollo sustentable. Esto ha implicado la adopción de convenciones marco y sus subsecuentes protocolos. Junto con implicar relaciones interestatales, estos instrumentos afectan las actividades de entes no estatales. A su vez, la cuestión aún pendiente de la responsabilidad por daño ambiental causada por actividades lícitas, es también uno de los temas que debe ser cuidadosamente considerado por la comunidad jurídica a fin de satisfacer simultáneamente los requerimientos de la soberanía en materia de desarrollo y la protección de valores ambientales.

En este mismo sentido, cabe reconocer las limitaciones y debilidades de los enfoques puramente jurídicos como base de la integración y cooperación económica. Una tendencia evidente en favor de instrumentos detallados celebrados en diversos períodos pasados, ha sido criticada y superada por la propia experiencia. La consicleración entre armonización de políticas económicas y la coordinación de sus instrumentos, está influyendo en el presente proceso de establecimiento de entidades y organizaciones con capacidad de decisión en la esfera económica a nivel internacional.

\section{Alcances de estos fenómenos en la enseñanza del Derecho Internacional}

La enseñanza y la investigación en Derecho Internacional, constituye parte del proceso de creación e interpretación del Derecho Internacional. No coincide necesariamente con el contenido actual del Derecho Internacional, pero contribuye a su conocimiento y difusión entre quienes se dedicarán a los asuntos jurídicos o participarán en la adopción de decisiones con relevancia jurídica.

Sin entrar en los tecnicismos de los estudios de Derecho, debe formularse una distinción entre la enseñanza a estudiantes de Derecho y la que se ofrece en programas dedicados a los estudios internacionales en un área más amplia.

\footnotetext{
${ }^{10}$ Tratado entre la República de Chile y la República Argentina sobre Promoción y Política Recíproca de Inversiones, 1991, aprobación en trámite.
} 
En el primer caso, uno de los objetivos principales de un programa de Derecho Internacional es introducir al estudiante en el conocimiento del ordenamiento jurídico internacional, sus sujetos y la atribución de competencias. Un profesor debe guiar con la adecuada distinción entre la lex lata y la lege ferenda. Debe asimismo, advertir acerca del estado de evolución de un concepto jurídico, acerca del proceso de cristalización de un nuevo principio o el debilitamiento de uno previamente establecido. ${ }^{11}$ Esto es válido tanto para el principio del patrimonio común de la humanidad incorporado en la Convención de las Naciones Unidas sobre el Derecho del Mar en 1982 y en el régimen aplicable a la luna y otros cuerpos celestes (espacio exterior), como para las explicaciones acerca de los principios básicos que rigen las relaciones entre los Estados o la noción de crimen como fundamento de la responsabilidad internacional en un nivel más severo.

Por otra parte, el Derecho Internacional debe ser explicado a través de su relación con los derechos e instituciones internas de los Estados. Incluso en sociedades con una fuerte tradición dualista como la hispanoamericana, la respuesta de los derechos internos a la evolución del Derecho Internacional es creciente. Esto ha dado lugar a revisiones de principios constitucionales básicos que establecen la jerarquía entre el Derecho Internacional y el interno, aceptando explícitamente la superioridad del primero.

Junto a este ámbito, la audiencia más apta para la enseñanza del Derecho Internacional es la de los estudios internacionales y la formación diplomática. Su propósito es hacer apreciable los vínculos entre las relaciones internacionales y los aspectos jurídicos de un problema o situación, a fin de analizar en profundidad los escenarios posibles en que interactúa el fenómeno político o económico y el jurídico, así como sus eventuales desenlaces. Este es el interés de una materia como solución de controversias en sus métodos diplomáticos y jurisdiccionales o de las competencias de las organizaciones internacionales en áreas de tradicional jurisdicción doméstica de los Estados.

Un caso de la práctica chilena reciente muestra el interés y la dificultad de apreciar esta estrecha relación. El otorgamiento a fines

\footnotetext{
${ }^{11}$ Abi-Saab, G., "La réformulation des principes de la Charte et la transformation des structures juridiques de la communauté internationale", Mélanges Michel Virally, Paris, Editions A. Pedone, 1991, pp. 1-8.
} 
de 1991 en la sede de la misión diplomática en Moscú, de la condición de "huésped" al ex Jefe de Estado de la República Democrática Alemana, Erik Honecker, ${ }^{12}$ generó una serie de debates en lo concerniente al derecho aplicable a su estatus y acerca de la solución del problema. Uno de los aspectos más controvertidos fue la explicación del rol que podía jugar el Derecho Internacional subyacente en el caso, el cual excluía el ejercicio del derecho de asilo diplomático y el recurso a métodos jurisdiccionales a menos que todas las partes involucradas así lo consintieran.

Esta última reflexión lleva a plantear el tema de los contenidos de una enseñanza actual del Derecho Internacional. No se trata solamente de determinar una lista de temas o un método acerca de cómo abordar diferentes áreas, como los sujetos, las fuentes u otras. Junto a esto, debe además tenerse en cuenta el equilibrio entre la teoría y la práctica y una comprensión amplia de los fenómenos de creación y aplicación de los principios jurídicos y sus regulaciones.

En el pasado, las teorías acerca de los enfoques regionales, como fue el innovador concepto del Derecho Internacional Americano, ${ }^{13}$ recibió un amplio apoyo entre los especialistas. Asimismo, análisis teóricos propuestos en países desarrollados y en desarrollo han avanzado ideas acerca de diferentes enfoques hacia el Derecho Internacional de acuerdo con la jerarquía económica de los Estados. Así, una agenda del Tercer Mundo ${ }^{14}$ debería ser tenida en cuenta junto a una agenda del mundo desarrollado.

Sin perjuicio de estas visiones que permiten aclarar los métodos a través de los cuales se modela y cambia el Derecho Internacional, se debe responder a los requerimientos de capítulos básicos relativos a la independencia de los Estados, la integridad territorial, la autodeterminación de los pueblos, la jurisdicción territorial y personal, para comprender las relaciones jurídicas internacionales en su cabalidad.

Otro punto que debe tenerse presente es que el contenido del Derecho Internacional evoluciona junto a necesidades y valores que abren las fronteras de los Estados, como es el caso de los derechos humanos y fortalecen las relaciones económicas entre ellos, superan-

\footnotetext{
12"Declaración Oficial de la Cancillería", 28 de julio de 1992, La Segunda, 29 de julio de 1992. ${ }^{13}$ Le Droit Intemational Américain, (París: Pedone, 1910).

${ }^{14}$ Henkin, L., "International Law: Politics, Values and Functions", Collected Courses, Académie de Droit International, 216, 1989, IV, pp. 345-347.
} 
do las tensiones y contradicciones expuestas y no resueltas plenamente en el concepto de nuevo orden económico internacional.

La. selección de temas fundamentales para la introducción al Derecho. Internacional puede cubrir, en consecuencia, los siguientes ámbitos:

- El estudio de las fuentes y la determinación de los principios de derecho aplicables a diferentes situaciones, hechos y reclamaciones. En esta etapa, la creación jurídica plantea la interrogante acerca de la introducción apropiada de los principios generales

$\therefore$ del derecho y de los principios de Jus Cogens. El estudio de ésto último implica la cuestión de la jerarquía entre las fuentes y la superación del paradigma de la igualdad soberana, siempre presente en: el ordenamiento internacional.

Este último tema subraya el rol de las organizaciones y de los tribunales internacionales. Como lo señala Michel Virally, ${ }^{15}$ "el derecho imperativo ocupa evidentemente un lugar importante en una estrategia concebida para limitar la libertad de acción de los más poderosos por el derecho: la estrategia de los más débiles, que no pueden limitar a la potencia (solución tradicional del Derecho Internacional)".

- En un ámbito diferente, la emergencia de temas como la protección del medio ambiente, migraciones y refugiados, nuevas áreas de protección de derechos humanos, plantean el rol del consentimiento en la creación del derecho consuetudinario. Otro caso importante en este ámbito es la interacción entre consentimiento y costumbre en el surgimiento del nuevo Derecho del Mar y las iniciativas para ampliar la aceptación universal de la Convención en materia de instituciones aplicables al régimen de los fondos marinos y oceánicos.

En cuanto a la aplicación del Derecho Internacional, algunos aspectos parecen fundamentales en la comprensión de su alcance, la cuestión de la responsabilidad y la solución de controversias. Adicionalmente, el problema de las contramedidas y versiones internacionales relacionadas con el uso de la fuerza, necesitan ser colocadas en el contexto de los requerimientos de la seguridad colectiva.

No se trata solamente de determinar si las sanciones de naturaleza penal pueden ser ejecutadas o si la reparación es el enfoque más

${ }^{15}$ Virally, M. "Panorama du Droit International Contemporain", Collected Courses, Académie de Droit International, 183, 1983, V, p. 176. 
eficaz, sino además de asegurar la aplicación del Derecho Internacional dentro del foro doméstico y las reacciones legítimas que pueden adoptar los Estados individuales y la comunidad internacional frente a las obligaciones erga omnes.

La famosa teoría de George Scalle acerca del "desdoblamiento funcional"16 explica bien la forma cómo opera el Derecho Internacional. Sin embargo, no cubre todas las dificultades que se presentan cuando se trata de compatibilizar el desarrollo de las organizaciones internacionales y las limitaciones al principio de no intervención en el sentido contemporáneo de la soberanía.

Teniendo en cuenta las dificultades de plantear el tema del Derecho Internacional en los estudios internacionales, en un ámbito dominado por las políticas exteriores, parece apropiado presentar un cuadro comprensivo de los escenarios reales de las relaciones jurídicas internacionales. Estas cruzan las fronteras de los Estados creando un cuadro complejo de conflicto y competencias superpuestas y de esfuerzos de compatibilización.

En vez de recargar los estudios jurídicos con las anomalías del sistema internacional, mayor énfasis debería ponerse en lo que clásicamente se ha llamado el Derecho Internacional Privado o las relaciones jurídicas entre sujetos a través de las fronteras de los Estados. Uno de los elementos centrales de la teoría del Derecho del Desarrollo, en auge en los años setenta, basada principalmente en la derogación de la igualdad entre los Estados, sus principales sujetos, debería ser complementada con enfoques más amplios acerca de las relaciones entre otras entidades. Este es el caso de las negociaciones para alcanzar acuerdos de libre comercio dentro de los procesos más recientes en las relaciones económicas entre países latinoamericanos.

La expansión de las relaciones internacionales y la búsqueda de un Derecho Internacional más efectivo en el ámbito interno de los Estados, merecen enfatizar la necesidad de estos nuevos enfoques en la enseñanza de esta disciplina tanto a estudiantes de derecho como en materia de estudios internacionales. La disminución de la distancia entre la teoría y la práctica, es también una necesidad para este propósito.

\footnotetext{
${ }^{16}$ Scelle, G., "Règles Générales du Droit de la Paix", Collected Courses, Académie de Droit International, 46, 1933, III, P. 426.
} 